\title{
SOBRE A PRIMEIRA LEI DA TERMODINÂMICA
}

\author{
Ney Henrique Moreira e Adalberto Bono Maurizio Sacchi Bassi* \\ Instituto de Química, Universidade Estadual de Campinas, CP 6154, 13083-970 Campinas - SP
}

Recebido em 22/2/00; aceito em 14/3/01

\begin{abstract}
ON THE FIRST LAW OF TERMODYNAMICS. Entropy is a concept that has long stimulated human curiosity, resulting in an huge intelectual production. The same has not occurred for the first law of thermodynamics, perhaps because of its apparent obviousness. In this article the first law presentation, as displayed in most traditional physical chemistry textbooks, is criticized. An alternative view is suggested, in accordance with temporal thermodynamics. The time derivative local form of the second law is used to stress the entropy concept implications on the notion of internal energy.
\end{abstract}

Keywords: internal energy; first law, temporal thermodynamics; rational thermodynamics.

\section{INTRODUÇÃO}

Como qualquer outra ciência, a termodinâmica não nasceu atemporal. Suas raízes mais profundas são encontradas em trabalhos de Euler (1753), Black (1757), Lambert (1779) e Lavoisier (1784). No começo do século dezenove tem-se Biot (1804), Fourier (1808/22) e Laplace (1816/22). Entretanto o trabalho de Carnot $^{1}$, em 1824, pode ser considerado o marco inicial da termodinâmica. A partir de então a teoria recebeu várias (e em alguns casos erráticas ${ }^{2}$ ) contribuições, sendo modificada entre 1850 e 1854 através da fundamental substituição da teoria calórica pelo conceito de que calor e trabalho são uniforme e universalmente interconversíveis, conforme experimentalmente demonstrado por Joule em 1847. Costumase denominar termodinâmica clássica à teoria que Clausius, Kelvin e Rankine edificaram entre 1850 e 1854, acrescida de trabalhos publicados posteriormente, especialmente por Clausius em 1862 (sua famosa desigualdade).

A teoria chamada clássica, portanto, é temporal e antecede à tendência estática, que se inicia imediatamente após. $\mathrm{O}$ tratado publicado por $\mathrm{Gibbs}^{3}$ em 1901 consolidou o já pré-existente enfoque estático, através dos lógicos conceitos estatísticos inicialmente desenvolvidos por Maxwell (publicados entre 1860 e 1879) e por Boltzman (publicados entre 1868 e 1896). A termodinâmica foi assim modernizada, adquirindo um novo brilho ao ser relacionada ao mundo microscópico que começava a atrair toda a atenção científica no começo do século vinte. Porém, entre outros, desde a sua defesa de tese de doutorado junto à universidade de Munique, em 1879, Planck não aceitou a idéia de que para ser matematicamente exata a termodinâmica devesse limitar-se a sua parte estática. Autor, desde 1891, de um dos mais famosos tratados sobre termodinâmica ${ }^{4}$, até sua morte, em 1947, Planck manteve sua convicção ${ }^{5}$.

Deve-se ressaltar que, ao contrário de outros que também optaram pelo enfoque estático (por exemplo, mais de duas décadas antes Kirchhoff e o próprio Gibbs, embora sem utilizar estatística, já defendiam tal ponto de vista), Gibbs foi quem conseguiu consolidá-lo não apenas por causa da atualização que a inclusão do comportamento das partículas trouxe à termodinâmica, mas principalmente porque ele foi um dos poucos a aplicar total rigor matemático ao estudo da termodinâmica (oito anos após, sem usar nem estatística nem ciclos de Carnot, mas sim a teoria para equações diferenciais de Pfaf, outra defesa da tendência estática, matematicamente bem fundamentada, foi publicada por Carathéodory ${ }^{6}$ ). Talvez por causa desta razão histórica, atualmente tende-se a usar lógica matemática rigorosa somente no tratamento estatístico da termodinâmica.
Em consequiência, muitos químicos consideram que este tratamento seja o único realmente seguro.

Entretanto, já foi elaborada uma teoria que, embora sendo completamente independente de valores estatisticamente esperados para sua exata compreensão lógica ${ }^{7-12}$, mantém coerência com as teorias estatísticas que se mostram capazes de produzir valores esperados concordantes com aqueles da mecânica dos meios contínuos ${ }^{13,14}$ (evidentemente a parte estática desta teoria mantém concordância com a termodinâmica estatística gibbsiana). Ela é a temporal termodinâmica dos meios contínuos, ativa área de pesquisas ${ }^{12,14}$ que faz a inatural separação entre termodinâmica e cinética química de imediato desaparecer, o que permite uma visão unificada e muito mais abrangente dos processos químicos.

Em especial, ressalte-se que a primeira lei é, de acordo com a termodinâmica dos meios contínuos, considerada um balanceamento de energia que, junto com as equações para balanceamento de massa e de momentos angular e linear, formam o coração da mecânica clássica, uma ciência temporal. Essa afirmação não corresponde apenas a uma razoável suposição conceitual. Na verdade, foi matematicamente demonstrado que os conceitos clássicos de espaço e tempo implicam em que a energia total de qualquer meio contínuo separa-se numa parte interna e outra cinética, o que produz como conseqüência a primeira $1 \mathrm{ei}^{15}$

Pretende-se neste trabalho apresentar aspectos inerentes à interpretação da definição matemática de energia interna, criticar apresentações da primeira lei freqüentemente encontradas e sugerir a alternativa oferecida pela termodinâmica dos meios contínuos. Sua intenção é colaborar na importante tarefa de aumentar a abrangência da termodinâmica, sem recorrer $a$ métodos estatísticos. O mesmo poderia ser feito em relação a segunda lei e, também, a todo embasamento da termodinâmica química, sem mudar absolutamente em nada as já bem estabelecidas e úteis aplicações práticas desta última, pelo contrário, potencialmente ampliando-as.

\section{DIFERENCIAIS E INTERVALOS FINITOS}

Em livros didáticos usuais, geralmente encontra-se a definição matemática da variação da energia interna de um sistema fechado,

$\Delta U=Q+W$

Esta equação limita-se a mostrar que a soma do calor com o trabalho trocados entre o sistema e seu exterior, durante um processo que ocorra entre estados de equilíbrio termodinâmico 
terminais, é igual à diferença entre as energias internas respectivamente associadas a estes dois estados, não fornecendo nenhuma informação, ou restrição, quanto aos estados intermediários que constituem o processo, logo sendo aplicável tanto para processos reversíveis como irreversíveis (independentemente dos significados que cada autor dê a estes adjetivos). Deve-se, entretanto, deixar claro que a energia cinética do sistema, tomado como corpo rígido, foi previamente subtraída do trabalho total.

Porém, é muitíssimo comum que, mantendo sua validade tanto para processos reversíveis como irreversíveis, a equação (1) seja indevidamente extrapolada para

$d U=d Q+d W$

Suponha-se $Y=y(X)$ uma função diferenciável, com derivada

$\mathrm{Y}^{\prime}=\mathrm{y}^{\prime}(\mathrm{X})=\frac{\mathrm{dY}}{\mathrm{dX}}, \log \mathrm{d} \mathrm{Y}=y^{\prime}(X) d X$. Lembre-se que $Y^{\prime}$ é o valor, bem determinado, da razão entre dois zeros, porque $\mathrm{Y}^{\prime}=\lim _{\Delta \mathrm{X} \rightarrow 0} \frac{\mathrm{dY}}{\mathrm{dX}}$. Lembre-se, também, que por definição um diferencial é livre para assumir qualquer valor real, mas somente valores reais (portanto $\pm \infty$ estão excluídos e zero está incluído), desde que tal real guarde, com o valor de pelo menos um outro diferencial, relação bem definida através de uma ou mais funções derivadas.

Ao se isolar dY não foi gerada incoerência matemática, pois os dois lados da equação foram multiplicados por $\mathrm{dX}$, o que mantém a integridade da relação. Por outro lado, a multiplicação dos dois lados da equação pelo inverso de $\mathrm{dX}$, ou de $\mathrm{dY}$, não poderia ser efetuada, porque $\mathrm{dX}$ e $\mathrm{dY}$ assumem, obrigatoriamente, também o valor zero e o inverso de zero diverge. Entretanto, se o valor zero pudesse ser proibido para $\mathrm{dX}$ e $\mathrm{dY}$, como de fato pode para $\Delta \mathrm{X}$ e $\Delta \mathrm{Y}$, tanto a multiplicação quanto a divisão por $\mathrm{dX}$ ou $\mathrm{dY}$ poderiam manter a integridade da relação. Por razões análogas, a igualdade (1) absolutamente não implica na (2), somente podendo ter significado matemático esta última se forem conhecidas, através das correspondentes funções derivadas, as definições dos três diferenciais envolvidos.

Estendendo a linha de raciocínio até aqui tomada conclui-se que, se $A=a(X, Z), B=b(X, Z)$ e se existir a equação diferencial $d Y=A d X+B d Z$, então ou $X=x(\Omega), Y=y(\Omega)$ e $\mathrm{Z}=\mathrm{z}(\Omega)$ existem e são diferenciáveis, ou $\mathrm{Y}=\mathrm{y}(\mathrm{X}, \mathrm{Z})$ existe e é diferenciável, ou ambas as condições são satisfeitas (nos dois últimos casos, a equação diferencial é denominada exata, sendo portanto inexata quando apenas a primeira condição ocorrer). Logo, se na equação (2) tiver$\operatorname{mos} d Q=T d S, \quad \mathrm{dW}=\sum_{\mathrm{j}=1}^{\mathrm{J}} \Phi_{\mathrm{j}} \mathrm{d} \Psi_{\mathrm{j}}, T=t\left(S, \Psi_{l}, \ldots, \Psi_{J}\right), \Phi_{j}=$ $\phi_{j}\left(S, \Psi_{l}, \ldots, \Psi_{J}\right) \quad$ para $\mathrm{j}=1, \ldots, \quad \mathrm{J}$ e $U=\left(S, \Psi_{l}, \ldots, \Psi_{J}\right)$ diferenciável, ela será uma equação diferencial exata, independentemente da denominação ou significado físico das variáveis e funções recém-propostas. Entretanto, se a equação (2) não for exata, ela não poderá ser postulada a menos que, conjuntamente, seus três diferenciais sejam definidos em termos de $\Omega$.

Note-se entretanto que: $(\alpha)$ a equação (2) costuma ser proposta antes de qualquer discussão sobre a segunda lei e $(\beta)$ se a única alteração na forma da apresentação fosse a reversão da ordem em que as duas leis são tradicionalmente propostas (persistindo, portanto, a omissão da definição dos diferenciais), a condição $d Q=T d S$ seria necessária para a validade da equação (2). Surge então, a interpretação de que $d U$ é um diferencial exato mas $d W$ e $d Q$ não são (sendo por causa disto em alguns livros ${ }^{16,17,18}$, mas não em todos os que adotam essa opinião ${ }^{19,20}$, até anotados através da substituição do símbolo " $d$ " por grafias que variam de um para outro texto), ou a suposição de que $d W$ e $d Q$ são intervalos finitos enquanto que $d U$ é um diferencial (neste caso omite-se o símbolo " $d$ " salvo antes da letra $U^{21,22}$ ).
De fato, equações diferenciais exatas e inexatas realmente existem conforme já afirmado, mas exclusivamente para o uso da visão estática da termodinâmica clássica criaram-se diferenciais exatos e inexatos ${ }^{10,23}$ e até desencontradas representações gráficas para estes últimos. Entretanto, mesmo que se deseje criar essa distinção, isto absolutamente não evita a exigência de que os diferenciais da equação inexata sejam definidos, sem o que ela perde seu significado matemático. Sob este aspecto tal criação é, portanto, completamente inócua. Por outro lado, a subtração de dois valores finitos positivos e bem determinados sempre produz um real bem definido (que evidentemente só será zero se os dois valores forem iguais), nunca um diferencial (que, conforme já lembrado, corresponde a um real indeterminado, o qual mantém uma relação bem definida com pelo menos outro real indeterminado).

Os livros didáticos sobre termodinâmica geralmente não apenas são, sob o aspecto matemático, profundamente diferentes daqueles de eletromagnetismo, mecânica, hidrodinâmica ou elasticidade por exemplo, como também da termodinâmica gibbsiana, tanto na sua parte estatística quanto naquela não estatística, esta última previamente publicada, durante décadas, com a mesma exatidão matemática característica de Gibbs. O contraste torna-se ainda mais intenso naqueles livros que, de acordo com a tendência talvez predominante entre químicos dedicados às áreas de mecânica quântica, espectroscopia ou termodinâmica estatística, enfocam a físico-química a partir da visão microscópica, para daí explicar o mundo macroscópico.

Estes últimos textos ${ }^{17,18}$ usam um linguajar necessariamente simplificado mas exato em suas respectivas partes iniciais, mas capitulam ao chegar, já na metade do livro ou após isso, à visão estática da termodinâmica clássica. Fato semelhante ocorre também em textos de mecânica de meios contínuos ou de ciência dos materiais destinados a físicos ou engenheiros, onde nos capítulos iniciais usam-se campos tensoriais, jacobianos etc., mas no capítulo de termodinâmica todo este arsenal é desprezado, optando-se por uma pseudo-matemática reservada exclusivamente para este capítulo.

\section{CONCLUSÕES CORRETAS MAS RESTRITAS}

Não se pense, entretanto, que as conclusões obtidas nos citados livros didáticos sejam falhas. Quando afirma-se que, não sendo satisfeita a condição $d Q=T d S$, a equação (2) só teria significado caso seus três diferenciais fossem definidos através de $\Omega$, isso não consiste em alguma sofisticação matemática, mas simplesmente num alerta de que, se tal definição não ocorrer, a equação se tornará completamente inútil $e$ portanto desnecessária. De fato, sempre que a equação diferencial (2) não for exata, usualmente apenas a equação (1) será utilizada, explicita ou implicitamente (neste último caso o livro usa a notação por ele convencionada para a equação (2), mas atinge somente os resultados obteníveis caso apenas a equação (1) existisse). Por outro lado, se (2) for uma equação diferencial exata, genericamente grafável sob a forma

$\mathrm{dU}=\mathrm{TdS}+\sum_{\mathrm{j}=1}^{\mathrm{J}} \Phi_{\mathrm{j}} \mathrm{d} \Psi_{\mathrm{j}}$

ela será plenamente utilizada, produzindo relações entre derivadas parciais etc...

Em alguns livros didáticos a equação (3) (às vezes acrescida em seu segundo membro do termo geralmente anotado $\sum_{\mathrm{j}=1}^{\mathrm{J}} \mu_{\mathrm{i}} \mathrm{dn}_{\mathrm{i}}$, referente a possíveis transformações químicas ou entre estados de agregação, o qual será nulo no equilíbrio químico e de fases) é considerada válida também quando a (2) não for exata mas, porque nesta situação as igualdades $d Q=T d S$ e $\mathrm{d} W=\sum_{\mathrm{j}=1}^{\mathrm{J}} \Phi_{\mathrm{j}} \mathrm{d} \Psi_{\mathrm{j}}$ serão falsas, em tal caso o segundo membro da expressão (3) não poderá mais ser subdividido em duas partes, 
sendo uma igual a $d Q$ e a outra igual a $d W$. Logo, a admissão de que a equação (3) seja válida em qualquer situação de um sistema fechado, sendo então denominada "equação fundamental" 20 , constitui-se num axioma não decorrente da (2) (nem da desigualdade de Clausius) e não representa o uso da equação (2) em situações nas quais $d Q=T d S$ seja falsa.

Portanto, se por primeira lei for basicamente entendido um balanceamento entre calor, trabalho e energia interna, a admissão de que a equação (3) continue válida também quando a (2) não seja exata absolutamente não corresponderia a uma ampliação na abrangência de tal lei. Cabe entretanto ressaltar que esta suposição apresenta fortíssimo significado conceitual, porque implica em que $U=u\left(S, \Psi_{1}, \ldots, \Psi_{j}\right)$ exista e seja diferenciável, logo implica em que $\mathrm{S}$ exista, independentemente da veracidade da igualdade $d Q=T d S$ (precisamente como queria Planck e a atual termodinâmica dos meios contínuos ${ }^{7-12}$ e antes dessa, também a termodinâmica do não equilíbrio ou dos processos irreversíveis ${ }^{24-29}$ ).

Porém a suspeita de que, semelhantemente à confusão já descrita entre as equações (1) e (2), alguns livros grafem " $d$ " quando na verdade interpretam " $\Delta$ ", tornaria absolutamente trivial, em tais livros, a afirmativa de que a equação (3) vale para qualquer processo. Aliás, substituindo-se " $d$ " por " $\Delta$ " a igualdade (3) somente teria validade se $T_{\text {inicial }}=T_{\text {final }} e$ $\Phi_{j \text { inicial }}=\Phi_{j \text { final }}$, sendo $j=1, \ldots, J$ o que a tornaria extremamente específica. Porque as conclusões obtidas nos livros didáticos geralmente não são falhas, a crítica ao modo como elas são conseguidas poderia até ser considerada inconveniente por quem julgasse que, num curso de química, não deveria haver preocupação com o conteúdo matemático. Absolutamente não pretendemos corrigir os livros didáticos usuais, mas sim colaborar na importante tarefa de ampliar a abrangência da termodinâmica, sem recorrer a métodos estatísticos (os quais, aliás, caracterizam-se pelo intenso apreço à exatidão matemática).

Entretanto, não há sequer como propor ampliação alguma, enquanto não for desfeita a ilusão de generalidade transmitida pelos livros didáticos usuais. No caso específico da primeira lei, antes de propor uma generalização da equação (2) para situações nas quais a igualdade $d Q=T d S$ seja falsa, é evidentemente necessário constatar que estes textos jamais usam a expressão (2) nestas condições e explicar que a razão disto reside na definição falha da mesma, o que impossibilita seu uso (embora estes mesmos textos afirmem a total generalidade dela). Em outras palavras, conforme antecipado na Introdução, os alicerces precisam ser alterados não para mudar qualquer uma das já bem conhecidas conclusões nestes livros apresentadas, mas sim para não permanecermos limitados somente a elas. Feitas as correções (que infelizmente não se restringem à primeira lei apenas), a temporal termodinâmica dos meios contínuos é, por exemplo, um imenso novo campo que se abre.

\section{AMPLIAÇ̃̃O PROPOSTA}

Para ampliar a abrangência da primeira lei, é necessário que os três diferenciais da equação (2) continuem definidos também quando ela não for exata. Para mostrar como isso pode ser obtido de forma fisicamente coerente, será inicialmente considerado um trabalho do qual a energia cinética do sistema, tomado como corpo rígido, tenha sido previamente subtraída. Sua expressão matemática será dada por $\mathrm{dW}=\sum_{\mathrm{j}=1}^{\mathrm{J}} \Phi_{\mathrm{j}} \mathrm{d} \Psi_{\mathrm{j}}$, onde $\Phi_{\mathrm{j}}$ é uma pressão generalizada (simétrico da pressão, ou potencial elétrico, ou tensão superficial etc.), enquanto que $\Psi_{\mathrm{j}}$ é um volume generalizado (respectivamente volume, ou carga elétrica, ou área superficial etc.). Fazendo $d W_{j}=\Phi_{j} d \Psi_{j}$, tem-se $\mathrm{W}_{\mathrm{j}}=\int \Phi_{\mathrm{j}} \mathrm{d} \psi_{\mathrm{j}}$.

Conforme amplamente conhecido, nesta última equação temse uma integral de linha, porque $\Phi_{j}$ não é função de $\Psi_{j}$ (a um volume não corresponde uma e somente uma pressão, a uma carga elétrica não corresponde um e somente um potencial etc.), portanto seja $\Phi_{j}$ como $\Psi_{j}$ devem ser definidos em função de um parâmetro comum. Conforme igualmente sabido, o único parâmetro clássico universal é o tempo $\Omega$ (p.e., a cada instante só pode corresponder um volume, uma pressão, um potencial, uma carga elétrica etc., embora a momentos diferentes possam corresponder o mesmo volume, ou carga etc., mas diferentes pressões, ou potenciais etc.).

A integral é então resolvida considerando-se $\Phi_{j}=\phi_{j}(\Omega)$, $\Psi_{j}=\psi_{j}(\Omega)$ diferenciável e $\mathrm{d} \Psi_{\mathrm{j}}=\frac{\mathrm{d} \Psi_{\mathrm{j}}}{\mathrm{d} \Omega} \mathrm{d} \Omega$, onde o diferencial foi definido através do valor $\frac{\mathrm{d} \Psi_{\mathrm{j}}}{\mathrm{d} \Omega}$ da função derivada. Os limites de integração serão $\Omega_{\text {inicial }}$ e $\Omega$, quando $\Omega$ for qualquer momento posterior a $\Omega_{\text {inicial}}$, o que indica que $\mathrm{W}_{j}=w_{j}(\Omega)$, logo $\mathrm{W}=w(\Omega)$, ou seja, que a cada instante posterior ao inicial corresponde um trabalho (independentemente de haver ou não equilíbrio termodinâmico em qualquer um dos dois instantes). Os livros didáticos de físico-química costumam escapar da integral de linha restringindo a apresentação a dois casos específicos: $(\alpha)$ existência da função $\Phi_{j}=\phi_{j}\left(\Psi_{j}\right)$ (p.e., trabalho expansivo isotérmico reversível de gás perfeito) e $(\beta)$ constância temporal de $\Phi_{j}$, quando basta conhecer a magnitude da variação de $\Psi_{j}$ entre os estados terminais (neste caso a função $\Phi_{j}=\phi_{j}(\Omega)$ foi fornecida e usada, embora de forma desapercebida).

Admitindo-se a conclusão experimentalmente obtida por Joule em 1847, de que calor e trabalho são uniforme e universalmente interconversíveis, o calor envolvido no processo também será uma integral de linha calculável exclusivamente através do uso do parâmetro tempo, a não ser em situações especialíssimas. Entretanto, o mesmo não ocorrerá com a soma de ambos, que de acordo com a equação (1) corresponde à variação da energia interna do sistema fechado, porque existe a possibilidade da equação diferencial (2) ser exata. Esta possibilidade pode ser exemplificada lembrando que, se $X$ e $Y$ forem variáveis independentes $\int \mathrm{XdY}$ e $\int \mathrm{YdX}$ somente poderão ser resolvidas quando fornecidas equações paramétricas para $X$ e $Y$, mas a obtenção de $\int(X d Y+Y d X)=\int d(X Y)=\Delta(X Y)$ exigirá apenas o conhecimento da magnitude da variação do produto $X Y=Z$, porque $\mathrm{dZ}=\mathrm{XdY}+\mathrm{YdX}$ é uma equação diferencial exata.

Entretanto, é evidente que se $W=w(\Omega)$ e $Q=q(\Omega)$ existirem e forem diferenciáveis, como de fato sempre são, de acordo com a equação (1) obrigatoriamente $U=u(\Omega)$ também existirá e será diferenciável. Em outras palavras, a primeira lei da termodinâmica pode ser escrita

$\frac{\mathrm{dU}}{\mathrm{d} \Omega}=\frac{\mathrm{dQ}}{\mathrm{d} \Omega}+\frac{\mathrm{dW}}{\mathrm{d} \Omega}$

A equação (4) é válida mesmo que a (2) não seja exata, portanto mesmo que os diferenciais desta última não possam ser determinados através de comparação com a (3). Aliás, neste último caso a igualdade (2) será inexata e seus diferenciais somente poderão ser definidos por comparação com a (4), ou seja,

considerando $\mathrm{d} U=\frac{\mathrm{d} U}{\mathrm{~d} \Omega} \mathrm{d} \Omega, \mathrm{dQ}=\frac{\mathrm{dQ}}{\mathrm{d} \Omega} \mathrm{d} \Omega$ e $\mathrm{dW}=\frac{\mathrm{dW}}{\mathrm{d} \Omega} \mathrm{d} \Omega$.

Em resumo, qualquer que seja a circunstância a equação (4) existe e, através dela, a igualdade (2), que por integração temporal produz a (1). Por outro lado, se considerarmos $U=u\left(S, \Psi_{l}, \ldots, \Psi_{J}\right)$ verdadeira apenas quando $d Q=T d S$, ou se postularmos que sempre exista, o mesmo acontecerá com (3), mas nos dois casos essa última implicará em (2) somente se $d Q=T d S$ e $\mathrm{dW}=\sum_{\mathrm{j}=1}^{\mathrm{J}} \Phi_{\mathrm{j}} \mathrm{d} \Psi_{\mathrm{j}}$. Se for suposto que a equação (2) seja válida em qualquer situação, então a expressão mais geral da primeira lei da termodinâmica será a equação (4). Esta última, aliás, é análoga aos temporais balanceamentos de massa e de momentos linear e angular da teoria mecânica. 


\section{CONCLUSÃO}

Quando, em cinética química, formula-se a lei de velocidade de uma reação, todas as grandezas intensivas são imaginadas espacialmente constantes no meio reacional, mas temporalmente variantes, situação esta que pode ser aproximada através de uma velocidade de homogeneização sensivelmente superior à de avanço da reação. A lei de velocidade, portanto, refere-se a um processo limite denominado homogêneo, no qual todas as variáveis (temperatura, pressão, concentrações, grau de avanço da reação, volume etc.) são funções do tempo. Entretanto, apenas num processo entre estados todos eles no equilíbrio químico, ou com o mesmo grau de avanço, pode-se ter $d Q=T d S$, logo em processos reacionais homogêneos geralmente a equação (2) não é aplicável, a não ser que seus diferenciais sejam definidos através de derivadas temporais, o que é análogo a usar a equação (4).

A proposta generalização da primeira lei rompe a barreira que isola a termodinâmica de todas as outras ciências naturais, entre elas a cinética química, abrindo caminho em direção aos processos heterogêneos, estudados pela termodinâmica dos meios contínuos. Evidentemente, porém, nem sequer a termodinâmica dos processos homogêneos, que é a porta de entrada para a dos meios contínuos, estará completa enquanto a segunda lei não for, também, expressa de forma temporal, o que impede o prosseguimento do exemplo de tratamento termodinâmico à cinética química iniciado no parágrafo anterior. $\mathrm{O}$ estudo da segunda lei, porém, ultrapassa a intenção deste trabalho. Note-se que a generalização proposta permite que o balanceamento de energia seja instantaneamente aplicado a qualquer processo, não apenas a processos homogêneos, enquanto que a generalização da segunda lei para processos heterogêneos ainda é objetivo de pesquisa.

Num processo homogêneo específico, para o qual $d Q=T d S$ e $\mathrm{dW}=\sum_{\mathrm{j}=1}^{\mathrm{J}} \Phi_{\mathrm{j}} \mathrm{d} \Psi_{\mathrm{j}}$, a equação (4) não se faz necessária, constituindo-se apenas numa informação adicional. Porém, os livros didáticos geralmente não exploram o fato de que, nesta particular situação em que a validade da equação (3) não é polêmica, a energia interna assuma o papel de um potencial termodinâmico, conforme sabido desde $1853^{30}$. Isto mostra que, nestas condições, a função diferenciável $\mathrm{U}=\mathrm{u}\left(\mathrm{s}, \Psi_{1}, \ldots, \Psi_{\mathrm{J}}\right)$ é suficiente para determinar não apenas a equação (2), mas também todas as grandezas constitutivas (características do material) do sistema fechado.

Evidentemente, tal fato encerra grande significado físico, ao indicar que $U$ representa bem mais do que apenas uma energia necessária para efetuar um balanceamento. Entretanto, mesmo se a função $U=u\left(S, \Psi_{1}, \ldots, \Psi_{J}\right)$ continuar existindo quando $d Q=$ $T d S$ for falsa (o que evidentemente implica na existência de $S$ em tais condições), $U$ não será um potencial termodinâmico se a equação (2) não for exata. Em outras palavras, o fato da equação (2) ser ou não exata altera sensivelmente não apenas sua matemática, mas também o significado físico da energia interna.

A equação (4) afirma que a taxa de variação de energia interna de um sistema será, a cada instante, igual à soma das taxas de troca de calor e de trabalho que o sistema efetuar. Note-se que estas duas últimas taxas são de compreensão intuitiva, porque estão ligadas à percepção sensorial, ao contrário dos conceitos de calor e trabalho. Em outras palavras, o que percebe-se sensorialmente são as potências, ou quantidades de energia transferida por unidade de tempo, não as quantidades totais transferidas ao longo de um intervalo temporal. Portanto, a generalização proposta levou a grandezas mais primitivas do que as originais, fato estranho porque, em geral, uma teoria mais abrangente envolve conceitos mais abstratos. Tal fato reforça nossa impressão de que alguns antigos mas ainda controversos conceitos, incluídos na visão estática da termodinâmica clássica transmitida pelos livros didáticos, são completamente desnecessários e até mesmo prejudiciais ao entendimento da teoria.
Por outro lado, embora a termodinâmica dos meios contínuos, assim como a mecânica destes meios ${ }^{31}$, envolva análise matemática moderna ${ }^{32}$, a teoria dos processos homogêneos, que é a única necessária para fundamentar a termodinâmica química, utiliza apenas as noções mais elementares de cálculo. Por exemplo, no presente trabalho usaram-se exclusivamente os conceitos de derivada, diferencial e integral de Riemann. Não há como considerar tal matemática demasiadamente elaborada. Ela, apenas, não é instrumental mas sim conceitual, como toda a ciência deveria ser. Aliás, a aversão à matemática, ou a noção de sua inacessibilidade, infelizmente tão comuns entre químicos, provém precisamente do tratamento simultaneamente maquinal e confuso que, às vezes, lhe é dado pelos próprios docentes desta bela, simples e transparente disciplina.

A teoria dos processos homogêneos é, no nosso entender, bem mais fácil de ser compreendida do que os já referidos conceitos que, embora apresentados em livros didáticos, ainda são objetos de controvérsia, como por exemplo semi-estático, reversível etc. Aliás, deve-se ressaltar que o único aspecto polêmico, no presente trabalho, é a dúvida sobre a validade ou não da equação (3), quando a (2) for inexata. Trata-se de polêmica no mínimo centenária. Planck, a termodinâmica do não equilíbrio (ou dos processos irreversíveis) e a termodinâmica dos meios contínuos consideram-na válida, enquanto que a termoestática de Gibbs, aparentemente não. Quanto aos livros didáticos, depende não somente de qual é aquele considerado, mas de supormos que ele usa corretamente, ou não, o conceito de diferencial.

Entretanto, no presente trabalho tal polêmica aparece de forma periférica, porque ele enfoca a primeira lei ao invés da segunda. Ele foi, propositalmente, desenvolvido levando em conta ambas as possibilidades, não havendo preferência por nenhuma. No mais, foram apresentadas idéias absolutamente bem estabelecidas, conforme uma rápida consulta à bibliografia indicará, mas pouquíssimo divulgadas não apenas entre químicos, mas entre físicos e engenheiros também. Já faz mais de uma década, porém, que pelo menos um livro didático para engenharia química as utiliza ${ }^{23}$.

\section{REFERÊNCIAS}

1. Carnot, S.; Réflexions sur la Puissance Motrice du Feu et sur les Machines propes à Développer cette Puissance; Bachelier; Paris, 1824.

2. Truesdell, C. A.; The Tragicomical History of Thermodynamics: 1822-1854, vol. 4 de Studies in the History of Mathematics and Physical Sciences; Springer-Verlag; New York, 1980.

3. Gibbs, J. W.; The Collected Works of J. Willard Gibbs; Longman, Green; New York, 1928.

4. Planck, M. K. E. L.; Vorlesungen über Thermodynamik; De Gruyter; Berlin, 1922.

5. Planck, M. K. E. L.; Wissenschaftliche Selbstbiographie.; J. A. Barth; Leipzig, 1948.

6. Carathéodory, C.; Investigation into de Foundations of Thermodynamics. In The Second Law of Thermodynamics; Kestin, J., Ed.; Dowden, Hutchinson and Ross; Stroudsburg, 1976.

7. Truesdell, C. A.; Toupin, R.; The Classical Fields Theories. In Handbuch der Physik, Vol. III, Part 1; Flügge, Ed.; Springer-Verlag; Berlin, 1960.

8. Day W. A.; The Thermodynamics of Simple Materials with Fading Memory; Springer-Verlag; New York, 1972.

9. Owen D. R.; A First Course in the Mathematical Foundations of Thermodynamics; Springer-Verlag; New York, 1984.

10. Truesdell C. A.; Rational Thermodynamics; SpringerVerlag; New York, 1984.

11. Truesdell C. A.; Bharatha S.; The Concepts and Logic of Classical Thermodynamics as a Theory of Heat Engines - 
Rigorously Constructec upon the Foundations Laid by S. Carnot and F. Reech; Springer-Verlag; New York, 1988.

12. Silhavý M.; The Mechanics and Thermodynamics of Continuous Media; Springer-Verlag; Berlin, 1997.

13. Truesdell C. A.; Muncaster R. G.; Fundamentals of Maxwell's Kinetic Theory of Simple Monoatomic Gas Treated as Branch os Rational Mechanics; Academic Press; Boston, 1980.

14. Muller I.; Ruggeri T.; Rational Extended Thermodynamics; vol. 37 de Springer Tracts in Natural Philosophy; SpringerVerlag; New York, 1998.

15. J. Serrin; Space, Time and Energy. In Nonlinear Analysis and Continuum Mechanics Papers for the $65^{\text {th }}$. Birthday of James Serrin; Buttazo G.; Galdi G. P.; Lanconelli E.; Pucci P.; Eds.; Springer-Verlag; New York, 1998.

16. Lewis, G. N.; Randall, M.; Thermodynamics; Mc-GrawHill; New York, 1961.

17. Ross J.; Berry R. S.; Rice S. A.; Physical Chemistry; John Wiley; New York, 1980.

18. McQuarrie D. A.; Simon, J. D.; Physical Chemistry, a Molecular Approach; University Sciences Books; Sausalito, CA, 1997.

19. Moore, W. J.; Physical Chemistry; Prentice-Hall, New Jersey, 1972.
20. Atkins P. W.; Physical Chemistry; Oxford Press, Oxford, 1998.

21. Guggenheim, E. A.; Thermodynamics, an Advanced Treatment for Chemists and Physicists; Elsevier Science; Amsterdan, 1998.

22. McGlashan, M. L.; Chemical Thermodynamics; Academic Press; London, 1979.

23. Astarita, G.; Thermodynamics, an Advanced Textbook for Chemical Engineers; Plenum Press; New York, 1989.

24. Onsager, L.; Phys. Rev. (2) 1931, 37, 405.

25. Onsager, L.; Phys. Rev. (2) 1932, 38, 2265.

26. Casimir, H. B. G.; Reviews of Modern Physics 1945, 17, 343

27. Prigogine, I.; Defay, R.; Chemical Thermodynamics; Longmans, Green; London, 1954.

28. Miller D. G.; Chem. Rev. 1960, 60,15.

29. De Groot, S. R.; Mazur, P.; Non-Equilibrium Thermodynamics; North-Holland; Amsterdan, 1962.

30. Reech, F.; Journal de Mathématiques Pures et Appliquées 1853, 18, 357.

31. Smith, D. R.; An Introduction to Continuum Mechanics after Truesdell and Noll; Kluwer, Dordrecht; The Netherlands, 1993.

32. Kuttler, K. L.; Modern Analysis; Studies in Advanced Mathematics; CRC Press; Boston, 1998. 